\title{
Journal of Communications
}

\section{CONTENTS}

\section{Volume 9, Number 1, January 2014}

Research on Channel Estimation Algorithm in 60GHz System Based on 802.15.3c Standard

Wei Shi, Hao Zhang, Xinjie Wang, Jingjing Wang, and Hongjiao Zhang

A Channel Coordination Scheme for High Density Vehicular Ad-Hoc Networks Based on Time Division

Multiplexing

Yao Zhang, Licai Yang, Haiqing Liu, and Lei Wu

Multiple-Symbol Differential Detection for Differentially Encoded LDPC Coded Systems Yang Yu and Shiro Handa

A Survey of MAC Protocols Design Strategies and Techniques in Wireless Ad Hoc Networks

Amjad Ali, Wang Huiqiang, Lv Hongwu, and Xiaoming Chen

A Performance Study of Downlink Scheduling Algorithms in Wireless Broadband Networks

Kuokkwee Wee, Muhd. Hilmi B. A. H, Yit Yin Wee, and Nima Saed

Energy Efficient Segmentation-Link Strategies for Transparent IP over WDM Core Networks.....

Mohd Nazri Mohd Warip, Ivan Andonovic, Ivan Glesk, Phaklen Ehkan, Fairul Afzal Ahmad Fuad, and

Mohamed Elshaikh Elobaid Said Ahmed

High-speed Downhole Transmission System and Its Synchronization Algorithm Based on Optical OFDM.

Renze Luo, Yonghua Ge, and Jiao Yang

Extending TLS with Mutual Attestation for Platform Integrity Assurance

NorazahAbd Aziz, NurIzura Udzir, and Ramlan Mahmod

Restoration for High-Overlapped Image Sequence with Composite Motion Blurs

Li-hui Zou, Jinwu Li, and Aziguli Wulamu

Fuzzy-based Clustering of Web Services' Quality of Service: A Review

Mohd Hilmi Hasan, Jafreezal Jaafar, and Mohd Fadzil Hassan

Diamond: An Improved Fat-tree Architecture for Large-scale Data Centers

Yantao Sun, Jing Chen, Qiang Liu, and Weiwei Fang 